\title{
Enhancing common-mode suppression in microstrip differential lines by means of chirped and multi-tuned electromagnetic bandgaps (EBGs)
}

\author{
P. Vélez, M. Valero, L. Su, J. Naqui, J. Mata-Contreras, J. Bonache, and F. Martín \\ GEMMA/CIMITEC, Departament d'Enginyeria Electrònica \\ Universitat Autònoma de Barcelona, 08193 BELLATERRA (Barcelona), Spain.
}

\begin{abstract}
This paper presents common-mode suppressed microstrip differential lines based on chirped electromagnetic bandgaps (EBGs). To achieve common-mode suppression in a wide band, the common-mode impedance is modulated following a sinusoidal chirping, whereas the differential-mode impedance is kept unaltered, thus ensuring that the line is transparent for that mode. The result is a differential line exhibiting common-mode suppression in a band from $2.45 \mathrm{GHz}$ up to $7.37 \mathrm{GHz}$ centered at $5 \mathrm{GHz}$ (98.4\% bandwidth). As compared to previous works, based on single tuned or double tuned EBGs, the proposed technique is useful to achieve wider rejection bands for the common mode. Nevertheless, the authors also propose an alternative based on multi-tuned EBGs, in order to obtain a comparable fractional bandwidth. In both cases, the design procedure is simple, and the ground plane is kept unaltered.
\end{abstract}

KEYWORDS: Electromagnetic bandgaps (EBGs), microstrip technology, differential lines.

\section{CORRESPONDING AUTHOR}

\author{
Paris Vélez \\ Departament d'Enginyeria Electrònica \\ Universitat Autònoma de Barcelona \\ 08193 BELLATERRA (Barcelona) \\ Spain
}

Tel.: 34935813513

Fax.: 34935812600

E-mail: paris.velez@uab.cat 


\section{Introduction}

Differential lines are of interest in many applications (e.g., high speed digital circuits) for their higher immunity to noise, electromagnetic interference (EMI) and crosstalk, as compared to the single-ended counterparts. However, the suppression of the common-mode in such differential lines is a due, at least in the frequency region of interest, since there are several sources of common-mode noise. Many types of common-mode suppressed differential lines have been reported in the literature [1-8]. In most of these lines, the common-mode is suppressed by etching patterns in the ground plane. Such patterns act as common-mode rejection filters, but do not significantly affect the differential mode, i.e., being all-pass structures for this mode. Although efficient common-mode suppression has been demonstrated in most of the previously cited works, one of the main limitations concerns the fact that the ground plane is altered (etched), this being prohibitive in certain applications (for instance, if backside isolation is a due). We have recently reported a technique that solves this problem, by applying the concept of electromagnetic bandgap (EBG) to differential microstrip lines [9].

Electromagnetic bandgaps are one-dimensional, two-dimensional or three-dimensional periodic structures able to inhibit signal transmission in a certain band in the vicinity of the so-called Bragg frequency [10], and eventually in the vicinity of the harmonics of that frequency. In the framework of transmission lines, signal rejection has been achieved by several means [11-16], but for the interest in this work, let us highlight the periodic modulation of line width in singleended transmission lines [17]. This causes a periodic perturbation in both the characteristic impedance and the coupling coefficient, which effectively suppresses signal propagation in the vicinity of the Bragg frequency, corresponding to that frequency with a guided wavelength equal to twice the period of the perturbation $[18,19]$. This idea has been translated to the suppression of the common-mode in differential microstrip lines, by merely modulating (periodically) the common-mode impedance, whilst keeping the differential-mode impedance unaltered [9]. This technique has been found to be efficient and avoids backside etching. However, since the amplitude of the modulation is limited (due to technological constraints), common-mode rejection bandwidth and rejection level cannot be optimized simultaneously with a single tuned EBG structure. This limitation has been partially solved by cascading two EBGs with different periods [9] (double tuned), and in this paper, we propose another solution consisting in a chirped EBG for the common-mode impedance. Chirped-EBGs structures have been used as dispersive delay lines [20-22]. By continuously varying the period of the perturbation along the line, we achieve a wide rejection band covering the range of frequencies corresponding to such period variation. Finally, the authors reproduce the frequency response of the differential Chirped-EBG by means of a differential four stage multi-tuned EBG, obtaining a comparable fractional bandwidth with better differential insertion loss level.

\section{Design methodology}

The design methodology to obtain common-mode rejection in differential chirped-EBG transmission lines is based on the work reported in [22]. The idea is to apply a sinusoidal chirped modulation to the characteristic impedance for the common-mode $\left(Z_{0 e}\right)$ keeping, at the same time, the differential-mode characteristic impedance uniform and equal to the reference impedance of the ports $\left(Z_{0 o}=Z_{0}=50 \Omega\right)$, hence obtaining an all-pass structure for that mode. The common-mode propagation characteristics can be controlled through the impedance profile off $Z_{0 e}$ along the propagation direction, $z$. In a non-uniform microstrip differential line with continuously varying width $(W)$ and separation between strips $(S)$ 
following a linearly frequency chirped modulation function, the resonant Bragg coupling between the quasi-TEM mode and the counter propagating mode is ideally satisfied for each frequency in only one point of the device. Following the design procedure reported in [22] the modulation profile for the common-mode impedance can be defined as:

$$
Z_{0 e}(\mathrm{z})=Z_{0 e(\mathrm{z}=0)} e^{k \cdot A(\mathrm{z}) \cdot\left\{1+\sin \left[\left(\frac{2 \pi}{a_{0}}-C \cdot L\right) \cdot \mathrm{z}+C \cdot \mathrm{z}^{2}-\frac{\pi}{2}\right]\right\}}
$$

where $a_{0}$ is the main spatial period of the profile impedance that is related to the perturbation profile period to a given frequency $f_{0}$. The peak amplitude modulation $k$ establishes the maximum coupling value and therefore the maximum $Z_{0 e}$ values. The response of the differential chirped EBG can be smooth using a windowing function $A(\mathrm{z})$. The parameter $C$ takes into account the chirp coefficient that fixes the slope of the linear variation of the spatial period. The total length of the line is represented by $L$, that is an integer multiple number $(N)$ of $a_{0}$. Notice that the expression (1) satisfies $Z_{0 e}(\mathrm{z}) \geq Z_{0 e}(0)=Z_{0 e}(\mathrm{~L})=Z_{0 o}(\mathrm{z})=50 \Omega$, as required in order to obtain a proper matching at the ports. Notice that if we increase the amplitude modulation in (1), this directly affects the maximum implementable value of common mode characteristic impedance $\left(Z_{0 e}\right)$, which is delimited by the technology (minimum strip separation of $160 \mu \mathrm{m}$ working with our available milling machine).

Once the operation frequency $\left(f_{0}\right)$ and the maximum rejection bandwidth $(\Delta f)$ of operation for the common-mode have been fixed, first of all we need to calculate a set of design parameters, which can be summarize as [22]:

$$
\begin{aligned}
a_{0} & =\frac{c}{\left.2 f_{0} \cdot \sqrt{\varepsilon_{\text {eff }}}\right|_{z_{0}}} \\
C & =\frac{\left.4 \pi \cdot \varepsilon_{\text {eff }}\right|_{z_{0}}}{\psi \cdot c^{2}} \\
L & =\frac{c \cdot \mid \psi \cdot \Delta f}{2 \sqrt{\varepsilon_{\text {eff }} \mid}}
\end{aligned}
$$

where $\varepsilon_{\text {eff }}$ is the substrate effective dielectric constant for $Z_{0}=50 \Omega$ transmission line and $c$ is the speed of the light in free space. The parameter $\psi$ is defined as group-delay slope. Finally we need to choose some parameters as the window function $A(\mathrm{z})$ and the modulation amplitude $k$. Using a window function causes a bandwidth reduction, but this phenomenon can be inverted by using a higher value of $k$ or increasing the total length $(L)$ of the line.

Once the value of the impedance profile for each propagation mode (differential and common) has been determined, using (1) for the common-mode impedance and setting the differentialmode characteristic impedance to $Z_{0 o}=Z_{0}=50 \Omega$, we need to calculate the geometry of the differential chirped-EBG transmission line. To do that we use the transmission line calculator (i.e. Agilent Linecalc) to obtain the width $(W)$ and separation $(S)$ strip in coupled lines considering a certain substrate. In the next section, the authors apply this design method to obtain a chirped EBG differential line with wideband common-mode rejection. 


\section{Enhanced common-mode rejection in differential lines based on chirped-EBG modulation}

Let us consider the design of a differential chirped-EBG transmission line with common-mode rejection in a frequency range of $\Delta f=5 \mathrm{GHz}$, with a group-delay slope of $\Psi=-0.5 \mathrm{~ns} / \mathrm{GHz}$ centered at $f_{0}=5 \mathrm{GHz}$. The considered substrate parameters are those of the Rogers $4003 \mathrm{C}$ substrate, with thickness $h=0.81 \mathrm{~mm}$ and dielectric constant $\varepsilon_{r}=3.55\left(\varepsilon_{\text {eff }}=2.8\right)$. Considering the chosen substrate, the design parameters have been obtained by means of equations (2-4). These parameters are: $a_{0}=17.9 \mathrm{~mm}, C=-781.906 \mathrm{~m}^{-2}$, and $L \geq 21.48 \mathrm{~cm}$. In this case a Kaiser window has been chosen in order to preserve the maximum value for the common-mode characteristic impedance with amplitude of $k=0.42$. At last, the total number of periods has been set to $N=12$.

The next step is to calculate the transverse geometry (inferred from equation 1) for the commonmode characteristic impedance $\left(Z_{0 e}\right)$ along the propagation direction, $z$. In Fig. 1, we can see that the minimum and maximum values for the common-mode characteristic impedance along the propagation direction are $53 \Omega$ and $132 \Omega$, respectively. Notice that $Z_{0 e}(\mathrm{z}=0)$ and $Z_{0 e}(\mathrm{z}=\mathrm{L})$ are slightly different (higher) than the port reference $Z_{0}=50 \Omega$, since achieving this value would need a significant separation between the strips of coupled lines.

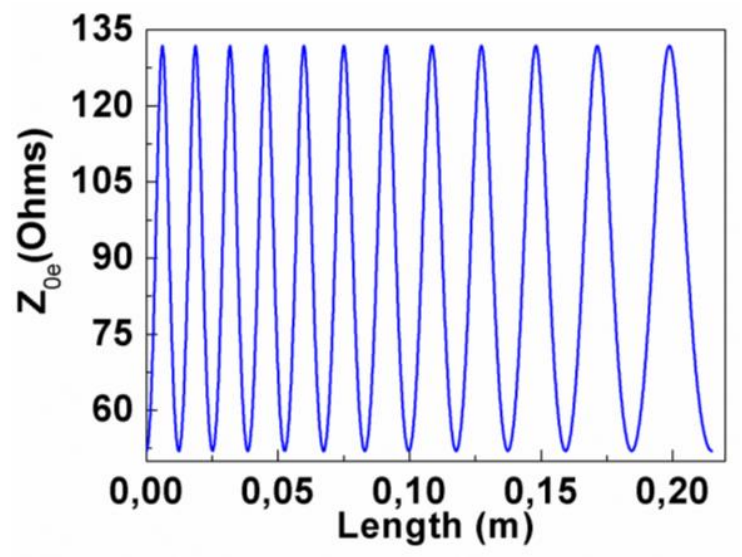

Fig. 1. Variation of the characteristic impedance for even mode with the propagation direction, $z$.

Since the transverse geometry is described by a continuous function along the chirped modulation, in practice this function has been discretized by 400 points and then the extremes of the different sections are interconnected obtaining a soft transition between them. The final layout is depicted in Fig. 2. Notice that input/output access lines are added, in order to have space for connector soldering and thus measure by means of a 4-port vector network analyzer.

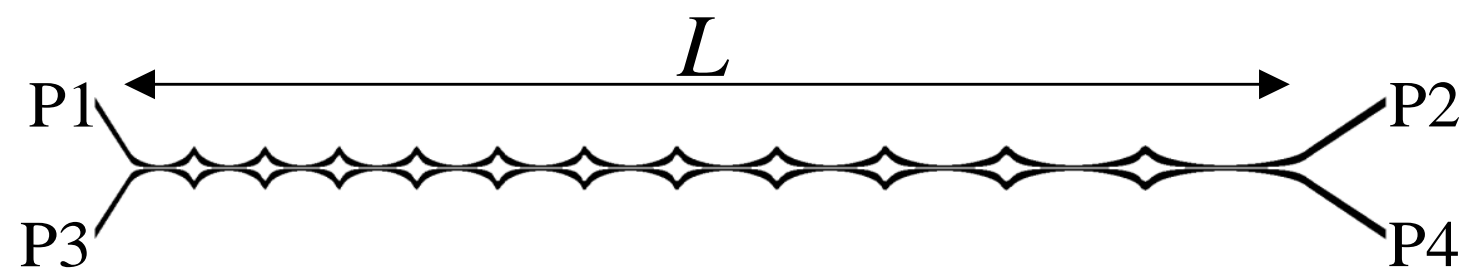

Fig. 2. Layout of the chirped-EBG common-mode suppressed differential line centered at $5 \mathrm{GHz}$. Device length is $L=22.1 \mathrm{~mm}$. 
The photograph of the fabricated differential line is shown in Fig. 3. Fig. 4 shows the lossless electromagnetic simulation (using Agilent Momentum 2011 software) and measured differential and common-mode insertion and return losses. Experimental data have been obtained by means of the 4-port Agilent PNA N5221A network analyzer. Both the differential and common-mode responses are in good agreement with the measurements up to roughly $10 \mathrm{GHz}\left(2 f_{0}\right)$. As (4) predicted, the results show a common-mode rejection bandwidth of $4.914 \mathrm{GHz}(98.2 \%)$, between the first common-mode reflections zeros near the maximum reflectivity. The all pass filter for the differential-mode has maximum insertion losses of $3.2 \mathrm{~dB}$ up to $8 \mathrm{GHz}$. At the central design frequency $\left(f_{0}=5 \mathrm{GHz}\right)$, the common-mode is rejected with $34.6 \mathrm{~dB}$ and the differential insertion loss is $1.6 \mathrm{~dB}$.

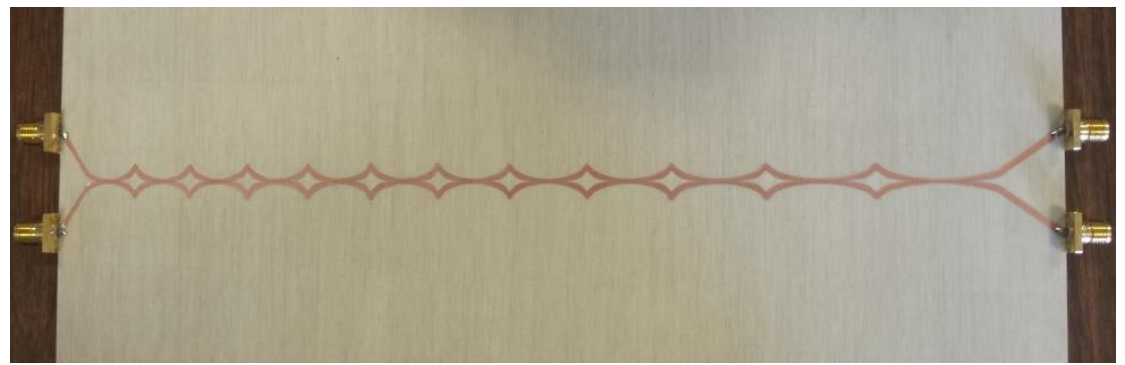

Fig. 3. Photograph of the $5 \mathrm{GHz}$ chirped-EBG based differential microstrip line with wideband common-mode rejection.
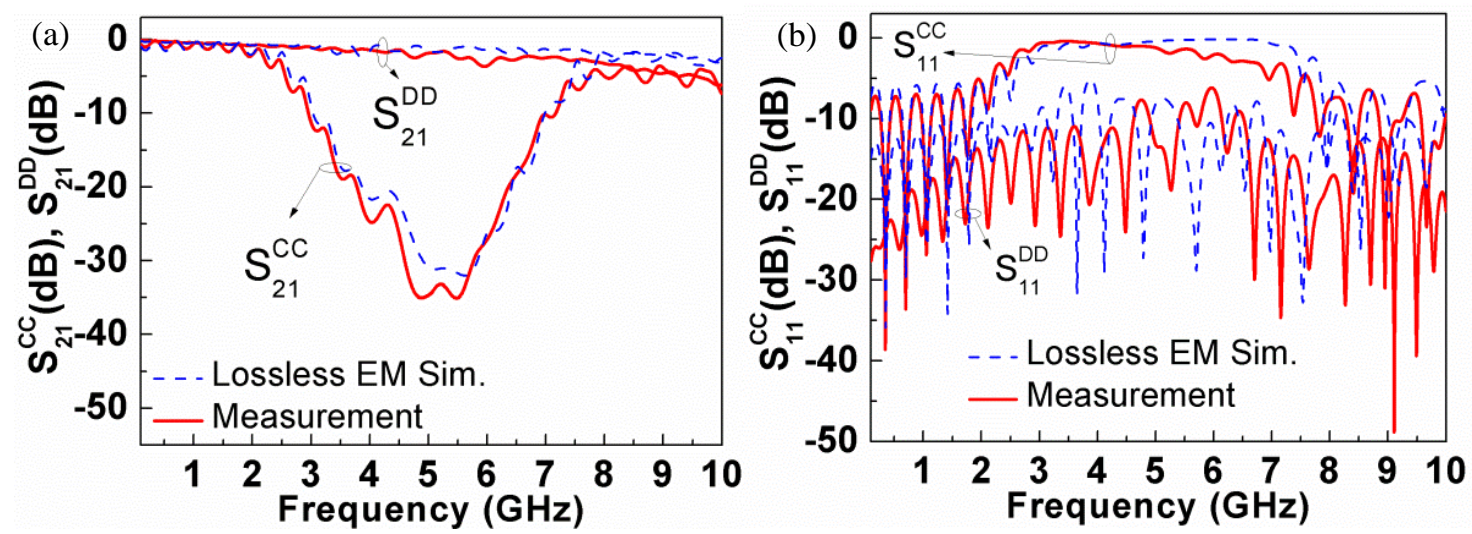

Fig. 4. Differential and common-mode insertion (a) and return (b) loss corresponding to the designed and fabricated chirped-EBG common-mode suppressed differential line of Figs. 2 and 3.

\section{Enhanced common-mode rejection in differential lines based on multi-tuned EBG}

As an alternative to the previous approach, the authors propose a strategy based on the multituned EBG concept developed in [9] in order to obtain a comparable frequency response. The main idea is to design four different EBG-based common-mode suppressed differential lines centered at frequencies covering the desired rejection band for the common-mode. Thus, following the procedure explained in [9], the common-mode characteristic impedance profile of each periodic EBG (considering a sinusoidal coupling coefficient in order to obtain a single rejection band [18] under common-mode excitation), is defined as

$$
Z_{0 e}(\mathrm{z})=Z_{0 e(\mathrm{z}=0)} e^{-\frac{2\left|K_{1}\right| \cdot \mid l}{\pi}\left[\cos \left(\frac{2 \pi}{l} z\right)-1\right]}
$$


where $K_{l}$ is the amplitude of the coupling coefficient and $l$ is the period that determines the frequency of maximum reflectivity according to the Bragg condition, i.e.:

$$
f_{\max }=\frac{c}{2 l \sqrt{\varepsilon_{\text {eff }}}}
$$

The maximum attenuation and rejection bandwidth for the common-mode are approximately given by [18]:

$$
\begin{gathered}
\left|S_{21}^{C C}\right|_{\min , n}=\operatorname{sech}\left(\left|K_{1}\right| \cdot L\right) \\
B W_{1}^{C C}=\frac{c\left|K_{1}\right|}{\pi \sqrt{\varepsilon_{\text {eff }}}} \sqrt{1+\left(\frac{\pi}{\left|K_{1}\right| \cdot L}\right)^{2}}
\end{gathered}
$$

where $L$ is the length of the structure (i.e., $L=l \cdot m, m$ being the number of cells), and the averaged effective dielectric constant can be calculated according to [19]:

$$
\overline{\varepsilon_{\text {eff }}}=\left(\frac{1}{l} \int_{0}^{l} \sqrt{\varepsilon_{\text {eff }}(z)} \cdot d z\right)^{2}
$$

Following the equations (5-9) we have designed four EBG-based differential lines with common-mode rejection of $14.9 \mathrm{~dB}$ at frequencies $f_{\max 1}=4 \mathrm{GHz}, f_{\max 2}=4.5 \mathrm{GHz}, f_{\max 3}=6 \mathrm{GHz}$ and $f_{\max 4}=7 \mathrm{GHz}$. The obtained bandwidths for the common-mode are: $80 \%\left(f_{\max }\right), 81 \%\left(f_{\max 2}\right)$, $80.8 \%\left(f_{\max 3}\right)$, and $81 \%\left(f_{\max 4}\right)$ with 3 cells $(m=3)$, and averaged effective dielectric constant of $\varepsilon_{\text {eff }}=2.9$. The parameters of these four periodic EBGs are summarized in Table I:

Table I. Element values for the EBGs centered at $4 \mathrm{GHz}, 4.5 \mathrm{GHz}, 6 \mathrm{GHz}$, and $7 \mathrm{GHz}$. The considered substrate is $R O 4003 C$ with thickness $h=0.81 \mathrm{~mm}$ and dielectric constant $\varepsilon_{r}=3.55$.

\begin{tabular}{c|c|c}
$\boldsymbol{f}_{\max }(\mathbf{G H z})$ & $\boldsymbol{L}(\mathbf{c m})$ & $\boldsymbol{K}\left(\mathbf{c m}^{-1}\right)$ \\
\hline 4 & 6.6 & 0.329 \\
\hline 4.5 & 5.79 & 0.307 \\
\hline 6 & 4.38 & 0.494 \\
\hline 7 & 3.75 & 0.576
\end{tabular}

Using the transmission line calculator, we can obtain the geometrical parameters for the EBGbased differential line, in order to reproduce the common-mode characteristic impedance profile of (5) at 40 discrete points for each line. The final layout for the multi-tuned differential EBG with common-mode suppression is shown in Fig. 5.

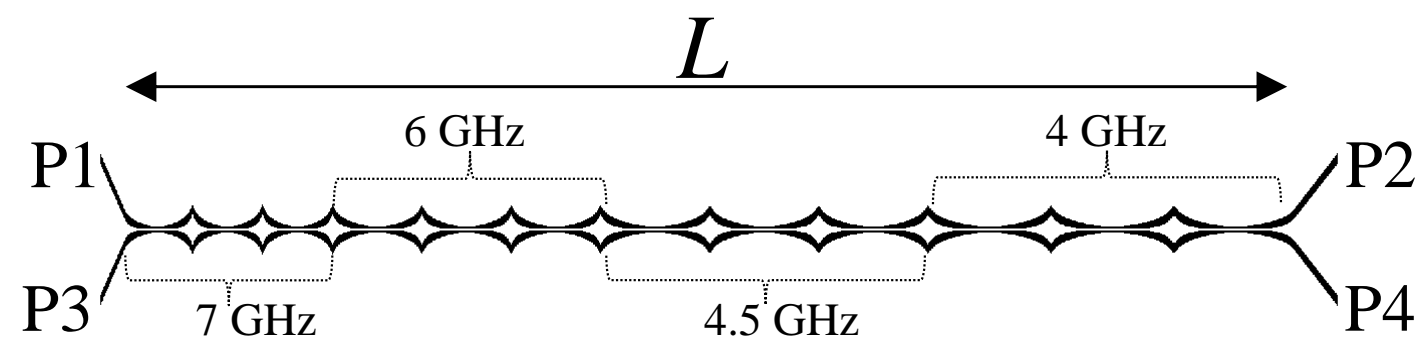

Fig. 5. Layout of the multi-tuned common-mode suppressed differential line centered at $5 \mathrm{GHz}$. Device length is $L=20.6 \mathrm{~mm}$.

The photograph of the fabricated common-mode rejection multi-tuned differential EBG is plotted in Fig. 6. In Fig. 7 we can observe that the agreement between the electromagnetic simulations (using Agilent Momentum 2011) and measurements (obtained by the 4-port 
Agilent PNA N5221A network analyzer) is good up to $10 \mathrm{GHz}$. In this case the common-mode rejection bandwidth is $5.1 \mathrm{GHz}(102 \%)$. The all-pass filter for the differential-mode has maximum insertion losses of $1.7 \mathrm{~dB}$ up to $9 \mathrm{GHz}$. At central design frequency $\left(f_{0}=5 \mathrm{GHz}\right)$, the common-mode is rejected with $34.6 \mathrm{~dB}$ (with maximum common-mode insertion loss of $39 \mathrm{~dB}$ at $4.7 \mathrm{GHz}$ ) and the differential insertion loss is $0.9 \mathrm{~dB}$. In order to compare the two approaches, we can observe in Fig. 8 the differential and common-mode insertion loss. In both cases we obtain a comparable size and common-mode fractional bandwidth rejection, but the multi-tuned EBG approach has better differential insertion loss.

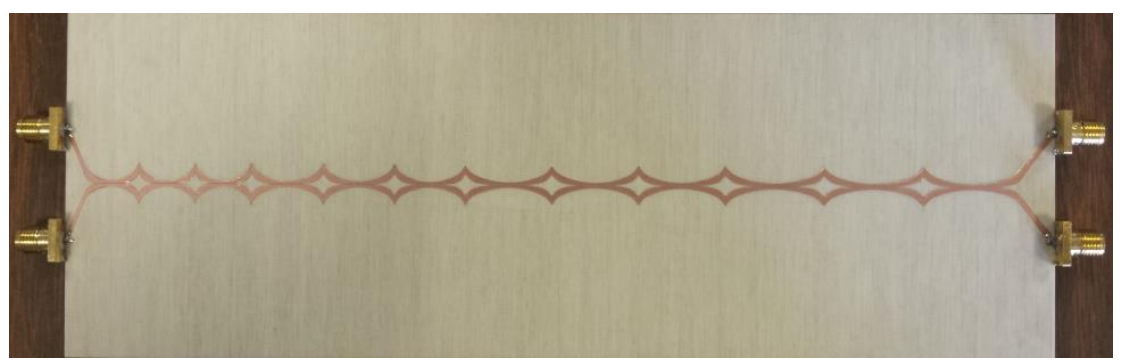

Fig. 6. Photograph of the $5 \mathrm{GHz}$ multi-tuned EBG-based differential microstrip line centered at $f_{0}=5 \mathrm{GHz}$.
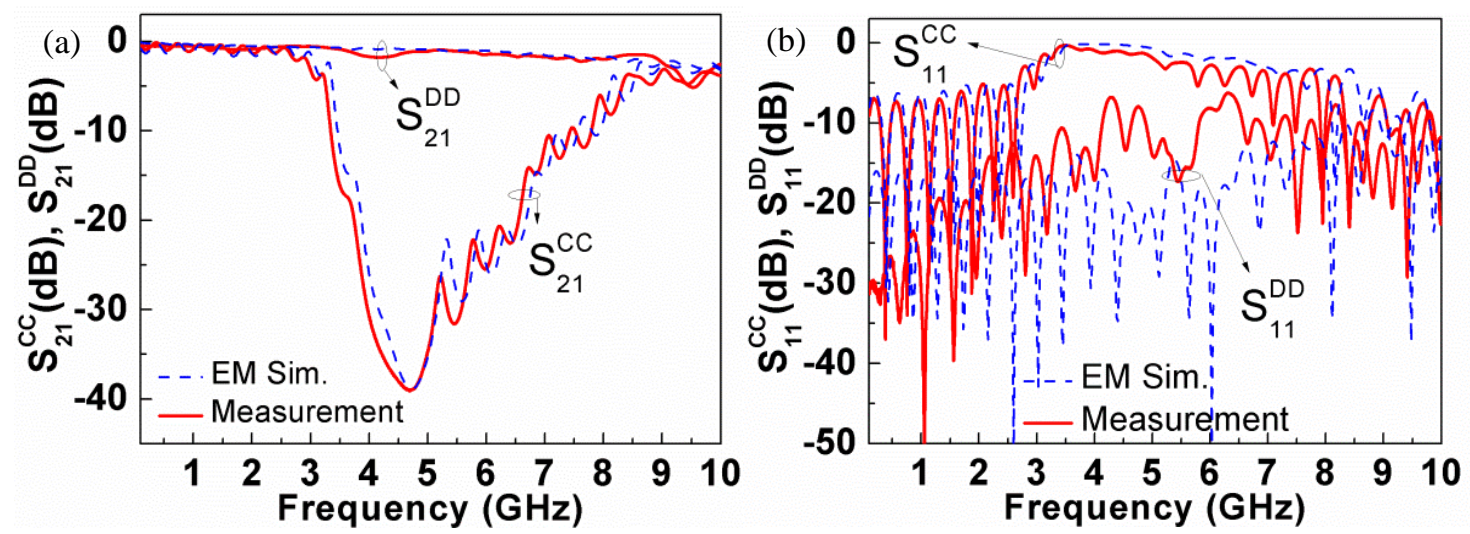

Fig. 7. Differential and common-mode insertion (a) and return (b) loss corresponding to the designed and fabricated multi-tuned differential EBG of Fig. 6.

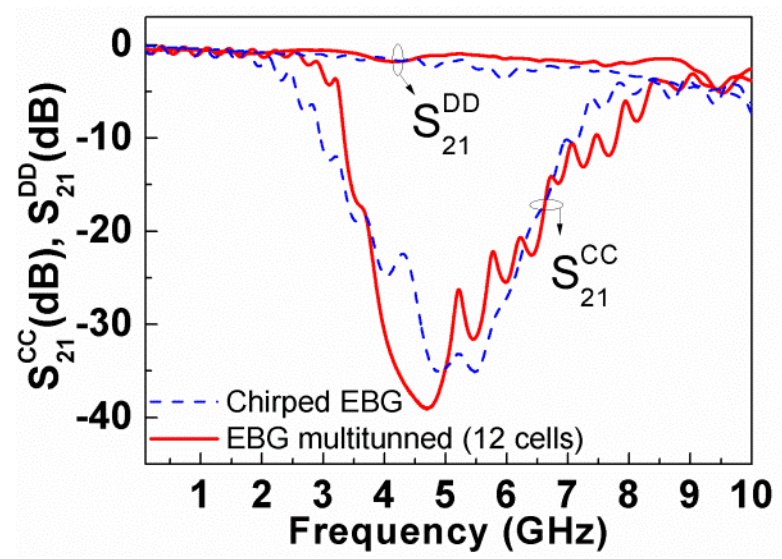

Fig. 8. Comparison between the measured insertion loss (for the two modes) of the two alternative designs reported in this work.

\section{Conclusions}

In conclusion, it has been shown that wideband common-mode suppression in differential lines can be achieved by using chirped EBGs or multi-tuned EBGs. The EBG impedance 
modulation has been applied only to the common-mode, keeping the differential-mode impedance unaltered. By this means, significant common-mode rejection has been achieved whilst the structure is all-pass to the differential mode. As compared to other reported approaches, the common-mode suppressed differential microstrip lines of this work do not include ground plane etching, this being an advantage (and even a due) in many applications.

\section{Acknowledgements}

This work was supported by MINECO-Spain (project TEC2013-40600-R), Generalitat de Catalunya (project 2014SGR-157), Institució Catalana de Recerca i Estudis Avançats (who awarded Ferran Martín), and by FEDER funds.

\section{References}

1. B. C. Tseng, and L. K. Wu, "Design of miniaturized common-mode filter by multilayer lowtemperature co-fired ceramic," IEEE Trans. Electromagn. Compat., vol. 46, no. 4, pp. 571-579, Nov. 2004.

2. C.-H. Tsai, and T.-L. Wu, "A broadband and miniaturized common-mode filter for gigahertz differential signals based on negative-permittivity metamaterials," IEEE Trans. Microw. Theory Techn., vol. 58, no. 1, pp. 195-202, Jan. 2010.

3. W.T. Liu, C.-H. Tsai, T.-W. Han, and T.-L. Wu, "An embedded common-mode suppression filter for $\mathrm{GHz}$ differential signals using periodic defected ground plane," IEEE Microw. Wireless Comp. Lett., vol. 18, no. 4, pp. 248-250, April 2008.

4. S.-J. Wu, C.-H. Tsai, T.-L. Wu, and T. Itoh, "A novel wideband common-mode suppression filter for gigahertz differential signals using coupled patterned ground structure," IEEE Trans. Microw. Theory Techn., vol. 57, no. 4, pp. 848-855, April 2009.

5. A. Fernández-Prieto, J. Martel, J. S. Hong, F. Medina, S. Qian, and F. Mesa, "Differential transmission line for common-mode suppression using double side MIC technology," Proc. 41st European Microwave Conference (EuMC), Manchester, UK, Oct. 2011, pp. 631-634.

6. J. Naqui, A. Fernández-Prieto, M. Durán-Sindreu, F. Mesa, J. Martel, F. Medina, and F. Martín, "Common mode suppression in microstrip differential lines by means of complementary split ring resonators: theory and applications", IEEE Trans. Microw. Theory Techn., vol. 60, pp. 3023-3034, Oct. 2012.

7. A. Fernandez-Prieto, J. Martel-Villagran, F. Medina, F. Mesa, S. Qian, J-.S Hong, J. Naqui, F. Martin, "Dual-band differential filter using broadband common-mode rejection artificial transmission line", Progress In Electromagnetics Research (PIER), vol. 139, pp. 779-797, 2013.

8. J. H. Choi, P. W. C. Hon, and T. Itoh, "Dispersion Analysis and Design of Planar Electromagnetic Bandgap Ground Plane for Broadband Common-Mode Suppression", IEEE Microw. Wireless Comp. Lett., vol. 24, pp. 772-774, Nov. 2014.

9. P. Vélez, J. Bonache, and F. Martín, "Differential Microstrip Lines with Common-Mode Suppression based on Electromagnetic Bandgaps (EBGs)", IEEE Ant. Wireless Propag. Lett., vol. 14, pp. 40-43, 2015.

10. E. Yablonovitch, "Photonic band gap structures", J. Opt. Soc. Amer. B 10, pp. 283-295, 1993.

11. V. Radisic, Y. Qian, R. Coccioli, T. Itoh, "Novel 2-D photonic bandgap structure for microstrip lines", IEEE Microw. Guided Wave Lett., vol. 8, pp. 69-71, 1998.

12. F. Falcone, T. Lopetegi, and M. Sorolla, "1-D and 2-D photonic bandgap microstrip structures", Microw. Opt. Techn. Lett., vol. 22, pp. 411- 412, 1999.

13. M. A. G. Laso, T. Lopetegi, M. J. Erro, D. Benito, M. J. Garde and Mario Sorolla, "Novel wideband photonic bandgap microstrip structures", Microw. Opt. Techn. Lett., vol. 24, pp. 357-360, 2000.

14. F. Falcone, T. Lopetegi, M. Irisarri, M. A. G. Laso, M. J. Erro, and M. Sorolla, "Compact photonic bandgap microstrip structures", Microw. Opt. Techn. Lett., vol 23, pp. 233-236, 1999.

15. M. A. G. Laso, T. Lopetegi, M. J. Erro, D. Benito, M. J. Garde, and M. Sorolla, "Multiplefrequency-tuned photonic bandgap microstrip structures", IEEE Microw. Guided Wave Lett., vol. 10, pp. 220-222, 2000.

16. T. Lopetegi, F. Falcone, and M. Sorolla, "Bragg reflectors and resonators in microstrip technology based on electromagnetic crystal structures", Int. J. Infrared Millimeter Waves, vol. 20, pp. 1091$1102,1999$. 
17. I. Arnedo, M. Chudzik, J. Schwartz, I. Arregui, A. Lujambio, F. Teberio, D. Benito, M.A.G. Laso, D. Plant, J. Azaña, and T. Lopetegi, "Analytical solution for the design of planar EBG structures with spurious-free frequency response", Microw. Opt. Techn. Lett., vol. 54, pp. 956-960, 2012.

18. T. Lopetegi, Photonic Band Gap Structures in Microstrip Technology: Study Using the Coupled Mode Formalism and Applications, PhD Thesis Dissertation, Pamplona, Spain, 2002.

19. F. Martin, Artificial Transmission Lines for RF and Microwave Applications, John Wiley, Hokoben (USA), 2015.

20. M. A. G. Laso, T. Lopetegi, M. J. Erro, D. Benito, M. J. Garde, M. A. Muriel, M. Sorolla, and M. Guglielmi, "Real-time spectrum analysis in microstrip technology", IEEE Trans. Microw. Theory Tech., vol. 51, pp. 705-717, 2003.

21. M. A. G. Laso, T. Lopetegi, M. J. Erro, D. Benito, M. J. Garde, M. A. Muriel, M. Sorolla, and M. Guglielmi, "Chirped delay lines in microstrip technology", IEEE Microw. Wireless Compon. Lett., vol. 11, pp. 486-488, 2001.

22. A. Lujambio, I. Arnedo, M. Chudzik, I. Arregui, T. Lopetegi, and M. A. G. Laso, "Dispersive delay line with effective transmission-type operation in coupled-line technology", IEEE Microw. Wireless Compon. Lett., vol. 21, pp. 459-461, 2011. 